\title{
Changes in gray matter volume and functional connectivity in dementia with Lewy bodies compared to Alzheimer's disease and normal aging: implications for fluctuations
}

\author{
Eléna Chabran ${ }^{1 *}$ (D), Vincent Noblet ${ }^{1}$, Paulo Loureiro de Sousa ${ }^{1}$, Catherine Demuynck ${ }^{2}$, Nathalie Philippi ${ }^{1,2}$,
} Catherine Mutter ${ }^{3}$, Pierre Anthony ${ }^{4}$, Catherine Martin-Hunyadi ${ }^{2}$, Benjamin Cretin ${ }^{1,2}$ and Frédéric Blanc ${ }^{1,2}$

\begin{abstract}
Background: Fluctuations are one of the core clinical features characterizing dementia with Lewy bodies (DLB). They represent a determining factor for its diagnosis and strongly impact the quality of life of patients and their caregivers. However, the neural correlates of this complex symptom remain poorly understood. This study aimed to investigate the structural and functional changes in DLB patients, compared to Alzheimer's disease (AD) patients and healthy elderly subjects, and their potential links with fluctuations.

Methods: Structural and resting-state functional MRI data were collected from 92 DLB patients, 70 AD patients, and 22 control subjects, who also underwent a detailed clinical examination including the Mayo Clinic Fluctuation Scale. Gray matter volume changes were analyzed using whole-brain voxel-based morphometry, and resting-state functional connectivity was investigated using a seed-based analysis, with regions of interest corresponding to the main nodes of the salience network (SN), frontoparietal network (FPN), dorsal attention network (DAN), and default mode network (DMN).

Results: At the structural level, fluctuation scores in DLB patients did not relate to the atrophy of insular, temporal, and frontal regions typically found in this pathology, but instead showed a weak correlation with more subtle volume reductions in different regions of the cholinergic system. At the functional level, the DLB group was characterized by a decreased connectivity within the SN and attentional networks, while the AD group showed decreases within the SN and DMN. In addition, higher fluctuation scores in DLB patients were correlated to a greater connectivity of the SN with the DAN and left thalamus, along with a decreased connectivity between the SN and DMN, and between the right thalamus and both the FPN and DMN.

(Continued on next page)
\end{abstract}

\footnotetext{
* Correspondence: elena.chabran@icube.unistra.fr

${ }^{1}$ ICube Laboratory UMR 7357 and FMTS (Fédération de Médecine

Translationnelle de Strasbourg), Team IMIS, University of Strasbourg and

CNRS, Strasbourg, France

Full list of author information is available at the end of the article
}

(c) The Author(s). 2020 Open Access This article is distributed under the terms of the Creative Commons Attribution 4.0 International License (http://creativecommons.org/licenses/by/4.0/), which permits unrestricted use, distribution, and reproduction in any medium, provided you give appropriate credit to the original author(s) and the source, provide a link to the Creative Commons license, and indicate if changes were made. The Creative Commons Public Domain Dedication waiver (http://creativecommons.org/publicdomain/zero/1.0/) applies to the data made available in this article, unless otherwise stated. 


\begin{abstract}
(Continued from previous page)
Conclusions: Functional connectivity changes, rather than significant gray matter loss, could play an important role in the emergence of fluctuations in DLB. Notably, fluctuations in DLB patients appeared to be related to a disturbed external functional connectivity of the SN, which may lead to less relevant transitions between different cognitive states in response to internal and environmental stimuli. Our results also suggest that the thalamus could be a key region for the occurrence of this symptom.
\end{abstract}

Keywords: Dementia with Lewy bodies, Fluctuations, MRI, Functional connectivity, Voxel-based morphometry, Salience network

\section{Background}

Dementia with Lewy bodies (DLB) is the second most common cognitive neurodegenerative disorder after Alzheimer's disease (AD), accounting for $15-20 \%$ of pathologically diagnosed dementia cases [1]. The core clinical features characterizing DLB, along with dementia, are cognitive fluctuations, recurrent visual hallucinations, rapid eye movement sleep behavior disorder (RBD) and spontaneous cardinal features of parkinsonism [1]. Of these symptoms, cognitive fluctuations appear to be both the most typical and the least understood. Defined as spontaneous alterations in cognition with pronounced variations in attention and alertness, they occur in $80-90 \%$ of DLB patients $[1,2]$ and have a significant impact on patients' autonomy and quality of life [3-5]. In daily life, they result in substantial changes in patients' mental status and behavior over time, alternating between periods of poorer task performance, incoherent speech and behavior, excessive daytime somnolence and episodes of altered consciousness (described by caregivers as sudden "switching off'), and periods of greater lucidity and cognitive performance $[1,6,7]$. Their periodicity and duration are highly variable even within the same person, ranging from seconds or minutes, to months. Clinically, fluctuations in DLB patients must be distinguished from the mild, day-to-day fluctuations commonly observed in various forms of dementia. They were shown to have a greater prevalence and severity than those observed in $\mathrm{AD}$ and vascular dementia $[2,7,8]$, and to be qualitatively distinct from fluctuations in $\mathrm{AD}$ patients, which rather consist in episodes of memory failure [9]. Furthermore, fluctuations in DLB patients seem to be internally driven, while those in $\mathrm{AD}$ patients may be more related to environmental triggers [9].

Despite fluctuations being a major symptom of DLB, their neural bases remain unclear. First, no consistent structural brain changes have been related to cognitive fluctuations, although a few studies have investigated this question. In a study combining diffusion tensor MRI and proton MR spectroscopy, Delli Pizzi et al. [10] reported a cholinergic imbalance in the right thalamus in DLB patients compared to controls and AD patients, along with microstructural alterations in bilateral thalamic regions projecting to the frontal cortex. However, only cholinergic measures correlated with the presence and severity of cognitive fluctuations, suggesting that neurochemical changes in this region could be more relevant to the physiopathology of fluctuations than microstructural alterations. More recently, significant atrophy was also found in the left pulvinar and ventral lateral nucleus regions of the thalamus in DLB patients and appeared to be associated with impaired attentional function [11]. Underlining the link between attentional impairment and cognitive fluctuations, the authors thus suggested a potential role of thalamic atrophy in the latter symptom. Lastly, a volumetric study focusing on the substantia innominata (SI) showed a significant negative association between the severity of cognitive fluctuations and gray matter volume in the right SI in DLB patients [12]. Overall, current findings do not allow clear conclusions on the structural correlates of this symptom but provide some clues towards a potential involvement of subcortical structures.

At the same time, an increasing focus is put on functional aspects, recent studies assuming that cognitive fluctuations are more likely to arise from functional network disturbances rather than overt structural abnormalities $[13,14]$. Electroencephalographic data showed that the frequency and severity of cognitive fluctuations were correlated with an increase and abnormal variability of posterior slow wave activity in DLB patients compared to AD patients [4, 15-17]. Similar posterior disturbances were observed using single-photon emission computed tomography (SPECT): cognitive fluctuations in DLB patients appeared to be associated with decreased inferior occipital perfusion and increased thalamic perfusion in one study [18] and with decreased perfusion in bilateral posterior parietal regions, covariant with increased perfusion in motor regions, in another [13]. In terms of functional connectivity, a significant association was found between greater fluctuations and a reduced connectivity between the right middle frontal gyrus and the right lateral parietal cortex in DLB patients [19]. Peraza et al. [14] also reported a positive correlation between alterations within the left frontoparietal network and the severity and frequency of cognitive fluctuations in DLB. According to these results, functional perturbations related to fluctuations thus seem to involve widely distributed networks rather than one particular brain region. 
The present study sought to complement and clarify current knowledge on the neural bases of fluctuations in DLB patients, by assessing both structural and functional aspects. Our aims were, first, to investigate changes in gray matter volume and functional connectivity in DLB patients compared to $\mathrm{AD}$ patients and healthy elderly subjects and, second, to assess more directly whether these structural and functional measures correlate with fluctuation scores in DLB patients. Since, in most of the previous studies, morphometric analyses were limited to a few a priori defined regions of interest, and functional connectivity analyses mainly focused on within-network changes, we aimed to provide a broader analysis framework by conducting our structural analysis on the whole brain and examining both within- and between-network functional connectivity in a set of well-described restingstate networks.

\section{Methods}

\section{Participants}

A total of 184 subjects participated in the study. Ninety-two DLB patients and $70 \mathrm{AD}$ patients were recruited through member teams of the Centre Mémoire de Ressources et de Recherche (CM2R) Alsace, France (i.e., the Geriatrics and Neurology Departments of the University Hospital of Strasbourg [HUS] and the Geriatrics Department of the General Hospital Center of Colmar). Additionally, 22 elderly healthy subjects were recruited through the Clinical Investigation Centre and CM2R of HUS. The study was approved by the Est-IV ethics committee (CPP Est-IV, Strasbourg), and all participants provided written informed consent.

To be included, the subjects had to be over the age of 50 years and be native speakers of French. Exclusion criteria were as follows: MRI contraindications, alcohol or substance abuse, sensory or motor disability, additional neurological or psychiatric conditions that could explain the symptoms, significant focal cerebral lesions shown on brain imaging, and co-occurrence of DLB and AD.

DLB and AD patients were diagnosed by experienced neurologists and geriatricians on the basis of the McKeith [2] and the Dubois [20] criteria, and the control participants were examined in the same way as patients to exclude any occult mild cognitive impairment or dementia cases. The potential presence of medial temporal atrophy was assessed using the Scheltens visual rating scale on brain MRI scans for the three groups. All participants underwent detailed clinical and neuropsychological evaluations, including the Mini-Mental State Examination (MMSE) and the Mayo Clinic Fluctuation Scale [6]. For a comprehensive description of the different neuropsychological assessments, see Kemp et al. [21].

We excluded 25 participants (13 DLB and $12 \mathrm{AD}$ patients) from the functional analysis for reasons of missing data $(n=12)$, image artifacts $(n=9)$, and to match the two dementia groups in terms of mean $\operatorname{MMSE}(n=4)$.

\section{Demographic and cognitive measures}

Statistical analyses of demographic and cognitive measures were performed using GraphPad Prism software (GraphPad Software, Inc., San Diego, CA). Normality of distribution of the variables was tested using the Shapiro-Wilk test and by visual inspection of quantile-quantile plots, and homogeneity of variances across groups was assessed using Levene's test. Where appropriate, between-group differences in continuous data were evaluated using either the non-parametric Kruskal-Wallis test with Dunn's post hoc test or a one-way ANOVA with Tukey's post hoc test. For categorical data, we used the chi-square test. Results were regarded as significant at $p<0.01$.

\section{MRI data acquisition}

Imaging was performed using a Siemens Verio 3T scanner equipped with a 32-channel head coil (Siemens, Erlangen, Germany). A concomitant resting-state blood oxygen level-dependent (BOLD) and arterial spin-labeling sequence was used to acquire 121 whole-brain T2*-weighted (gradient echo) echo planar images. The parameters were as follows: repetition time $=3 \mathrm{~s}$; flip angle $=90^{\circ}$; echo time $=21 \mathrm{~ms}$; inversion time $1=600 \mathrm{~ms}$; inversion time $2=1325.1 \mathrm{~ms}$; field of view $=152 \times 256 \times 112 \mathrm{~mm}$; 4-mm isotropic voxels. The first volume was intended for arterial spin-labeling measurement; thus, it was not considered for BOLD analysis. One whole-brain T1-weighted image was also collected within the same session, using a 3D magnetization-prepared rapid gradient-echo (MPRAGE) sequence. The parameters were as follows: repetition time $=1.9 \mathrm{~s}$; flip angle $=9^{\circ}$; echo time $=2.53 \mathrm{~ms}$; inversion time $=900 \mathrm{~ms}$; field of view $=192 \times 192 \times 176 \mathrm{~mm}$; $1-\mathrm{mm}$ isotropic voxels.

\section{MRI data preprocessing}

Images from each subject were preprocessed using the Statistical Parametric Mapping 12 package (SPM12, The Wellcome Trust Centre for Neuroimaging, London, UK).

\section{Structural images}

The anatomical data set was first visually inspected to check for potential artifacts or anatomical abnormalities and then spatially preprocessed using standard procedures [22]. All T1 structural images were segmented and bias corrected using an extension of the unified segmentation procedure [23] including six classes of tissue. The DARTEL approach was then used to build a studyspecific template and to spatially normalize all segmented images to the Montreal Neurological Institute (MNI) space. Lastly, gray matter images were modulated 
to preserve the total amount of gray matter from the original data and smoothed with a 8-mm full-width at halfmaximum Gaussian kernel.

\section{Functional images}

Functional images were preprocessed with the following steps: low-pass filtering at $0.112 \mathrm{~Hz}$ to remove arterial spin-labeling frequencies; slice-timing correction; rigid body registration and B0 field inhomogeneity correction; coregistration to the $\mathrm{T} 1$-weighted anatomical image; spatial normalization to Montreal Neurological Institute space using the DARTEL approach with an 8-mm fullwidth at half-maximum Gaussian kernel. No modulation was applied during the normalization procedure. We ensured that there were no between-group differences in the total amount of head motion (corresponding to the maximum absolute frame-wise displacement across scans), using ANOVA $(\mathrm{F}(2,156)=0.34, p=0.71)$. Finally, a denoising step using the aCompCor method [24] including cerebrospinal fluid, white matter, and motion parameters was conducted to remove residual unwanted motion and physiological and artefactual effects from the BOLD signal, prior to connectivity analyses.

\section{Statistical analysis \\ Voxel-based morphometry (VBM) analysis}

Group comparisons Between-group differences in gray matter volume were assessed using the SPM12 General Linear Model based on Gaussian random field theory [25]. Age, gender, and total intracranial volume were included in the design matrix as covariates of no interest. Statistical significance was set at a false discovery rate (FDR)-corrected threshold of $p<0.001$ at the voxel level.
Association between VBM measures and clinical variables $A$ multiple regression analysis was performed to examine the effect of gray matter volume on fluctuation scores in the DLB group separately. Variables entered into the model along with the dependent variable included the fluctuation scores, as a covariate of interest, and age, sex, and total intracranial volume, as covariates of no interest. Results were reported when significant at an uncorrected threshold of $p<0.05$ at the voxel level.

\section{Resting-state functional connectivity analysis}

ROI-to-ROI analysis Seed-based functional connectivity analyses were performed using the Conn toolbox [26]. Twenty-two regions of interest (ROIs) corresponding to the main nodes of the salience network (SN), frontoparietal network (FPN), dorsal attention network (DAN), and default mode network (DMN) were selected from the "networks atlas" implemented in the Conn toolbox, which was obtained from an independent component analysis (ICA) including 497 subjects from the Human Connectome Project dataset [26]. Additionally, two other ROIs were defined based on the results of the VBM analysis, corresponding to brain regions where gray matter volume was correlated to fluctuation scores in DLB patients. They were added in the connectivity analysis using the Harvard-Oxford Atlas [27] implemented in the Conn toolbox. See Fig. 1 and Additional file 1: Table S1.

At the first level of the analysis, individual ROI-to-ROI functional connectivity matrices were generated by computing bivariate Pearson's correlation measures between the mean BOLD signal time courses of each pair of ROIs. Each participant's motion parameters obtained during the preprocessing were added as a covariate of no interest. A Fisher transformation was then applied to

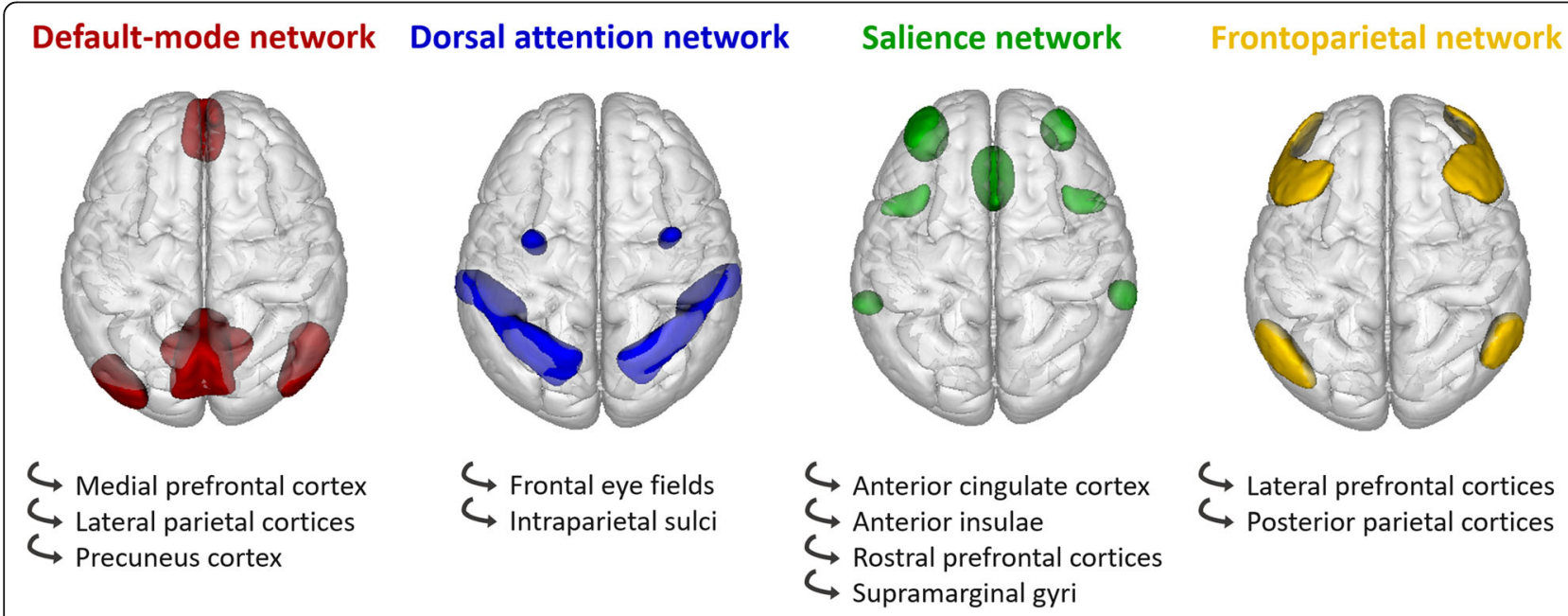

Fig. 1 Regions of interest used in the functional connectivity analysis, superimposed on a three-dimensional brain template (superior view) 
the correlation coefficients to improve normality assumptions of the subsequent, second-level analyses.

In a next step, the individual matrices were entered into a second-level general linear model, with age and gender as covariates of no interest, to allow betweengroup comparisons. The results were reported only when surviving a false discovery rate (FDR) corrected threshold of $p<0.05$ at the seed level.

Mean within- and between-network connectivity In addition, mean within-network and between-network connectivity values were computed in the second level of the analysis, to obtain more summary measures of the functional changes in the different groups of participants. The mean within-network connectivity was calculated as an average of the connectivity values between all ROIs within a network, while the mean between-network connectivity corresponded to an average of the connectivity values between all possible ROI pairs in two chosen networks.

Association between connectivity measures and clinical variables Multiple regression analyses were also performed to examine the effect of functional connectivity on fluctuation scores, in the DLB group separately. Variables entered into the model along with the dependent variable included the fluctuation scores as a covariate of interest, and age and gender as covariates of no interest. Results were reported when significant at an uncorrected threshold of $p<0.05$ at the seed level.

\section{Results}

\section{Demographic and cognitive measures}

Demographic and cognitive data of the participants are summarized in Table 1 . In the VBM cohort, the AD group was older than the control and DLB groups, while the latter were comparable in age. In the cohort used for the functional analysis (which was the same cohort as for the VBM analysis but with 25 fewer participants), the $\mathrm{AD}$ group was older than the control group but not the DLB group, and the latter were comparable in age. In both cohorts, the two dementia groups had lower MMSE scores than the control group, but did not differ from each other for this variable. As expected, the DLB group had higher fluctuation scores than both other groups. Finally, all groups were comparable for gender.

\section{VBM analysis}

Group comparisons

Compared with the control group, the AD group showed a widespread pattern of atrophy involving mainly the medial temporal lobe. The DLB group differed from the controls by showing more focal patterns of gray matter loss, involving the temporal lobes, the insulae, and the frontal lobes. When comparing the AD and DLB groups, we observed a greater gray matter loss in the medial temporal lobe (including the parahippocampal gyrus, the hippocampus and the amygdala) in AD patients. No regions showed greater atrophy in the control group than

Table 1 Demographics and fluctuation scores of the cohorts

\begin{tabular}{|c|c|c|c|c|}
\hline$A$ & $\mathrm{HC}(n=22)$ & $\operatorname{DLB}(n=92)$ & $\mathrm{AD}(n=70)$ & Between-group differences \\
\hline Gender (m/f) & $11 / 11$ & $39 / 40$ & $26 / 32$ & $x^{2}=0.42, p=0.81$ \\
\hline Age & $66.5 \pm 7.8$ & $70.1 \pm 9.4$ & $74.4 \pm 8.3$ & $\begin{array}{l}\text { AD vs HC: } p=0.0010 \\
\text { AD vs DLB: } p=0.0084 \\
\text { DLB vs HC: NS }\end{array}$ \\
\hline MMSE & $28.9 \pm 0.9$ & $25.6 \pm 4.0$ & $23.8 \pm 3.8$ & $\begin{array}{l}\text { DLB vs HC: } p=0.0001 \\
\text { AD vs HC: } p<0.0001 \\
\text { DLB vs AD: NS }\end{array}$ \\
\hline MCFS & $0.3 \pm 0.6$ & $1.9 \pm 1.2$ & $0.7 \pm 1.0$ & $\begin{array}{l}\text { DLB vs HC: } p<0.0001 \\
\text { DLB vs AD: } p<0.0001 \\
\text { AD vs HC: NS }\end{array}$ \\
\hline B & $\mathrm{HC}(n=22)$ & $\operatorname{DLB}(n=79)$ & $\operatorname{AD}(n=58)$ & Between-group differences \\
\hline Gender (m/f) & $11 / 11$ & $39 / 40$ & $26 / 32$ & $x^{2}=0.33, p=0.85$ \\
\hline Age & $66.5 \pm 7.8$ & $70.3 \pm 9.5$ & $73.7 \pm 8.3$ & $\begin{array}{l}\text { AD vs HC: } p=0.0045 \\
\text { DLB vs HC: NS } \\
\text { DLB vs AD: NS }\end{array}$ \\
\hline MMSE & $28.9 \pm 0.9$ & $25.8 \pm 3.8$ & $24.4 \pm 3.2$ & $\begin{array}{l}\text { DLB vs HC: } p=0.0002 \\
\text { AD vs HC: } p<0.0001 \\
\text { DLB vs AD: NS }\end{array}$ \\
\hline MCFS & $0.3 \pm 0.6$ & $1.8 \pm 1.2$ & $0.6 \pm 0.9$ & $\begin{array}{l}\text { DLB vs HC: } p<0.0001 \\
\text { DLB vs AD: } p<0.0001 \\
\text { AD vs HC: NS }\end{array}$ \\
\hline
\end{tabular}

(A) Cohort of the voxel-based morphometry analysis. (B) Cohort of the functional connectivity analysis. Data are presented as mean \pm SD except where noted. Abbreviations: AD Alzheimer's disease, DLB dementia with Lewy bodies, HC healthy controls, $f$ female, $m$ male, MCFS Mayo Clinic Fluctuation Scale, MMSE MiniMental State Examination, NS non-significant 
in the dementia groups, nor in the DLB group than in the AD group (see Fig. 2).

\section{Association between VBM measures and fluctuation scores}

In the DLB group (see Table 2), we found a negative correlation between patients' fluctuation scores and gray matter volume in clusters including the left inferior parietal lobule, the left and right cerebellum, the midbrain, the right middle orbitofrontal gyrus, and the left superior orbitofrontal gyrus. When raising the threshold to $p<$ 0.005 , this negative correlation also appeared in the right thalamus.

Among these regions, the midbrain and the thalamus were added as complementary ROIs for the subsequent functional connectivity analysis. The left inferior parietal gyrus and the prefrontal cortex did not need to be added as they already corresponded to nodes of the selected functional networks.
We did not find any cluster where gray matter volume was positively correlated to patients' fluctuation scores.

\section{Functional connectivity analysis ROI-to-ROI analysis}

First, the DLB group showed a significant decrease in functional connectivity between a number of ROIs within the $\mathrm{SN}$, compared to the control group (see Fig. 3). Similar decreases were found between ROIs within the FPN (right lateral prefrontal cortex [LPFC] and right posterior parietal cortex $[\mathrm{PPC}]$ ) and between ROIs of the FPN and the DAN (right LPFC and right intraparietal sulcus [IPS], respectively). In contrast, a significantly increased functional connectivity was observed between the medial prefrontal cortex (MPFC) of the DMN and both the right rostral prefrontal cortex (RPFC) of the SN and the right LPFC of the FPN, in DLB patients compared to controls.

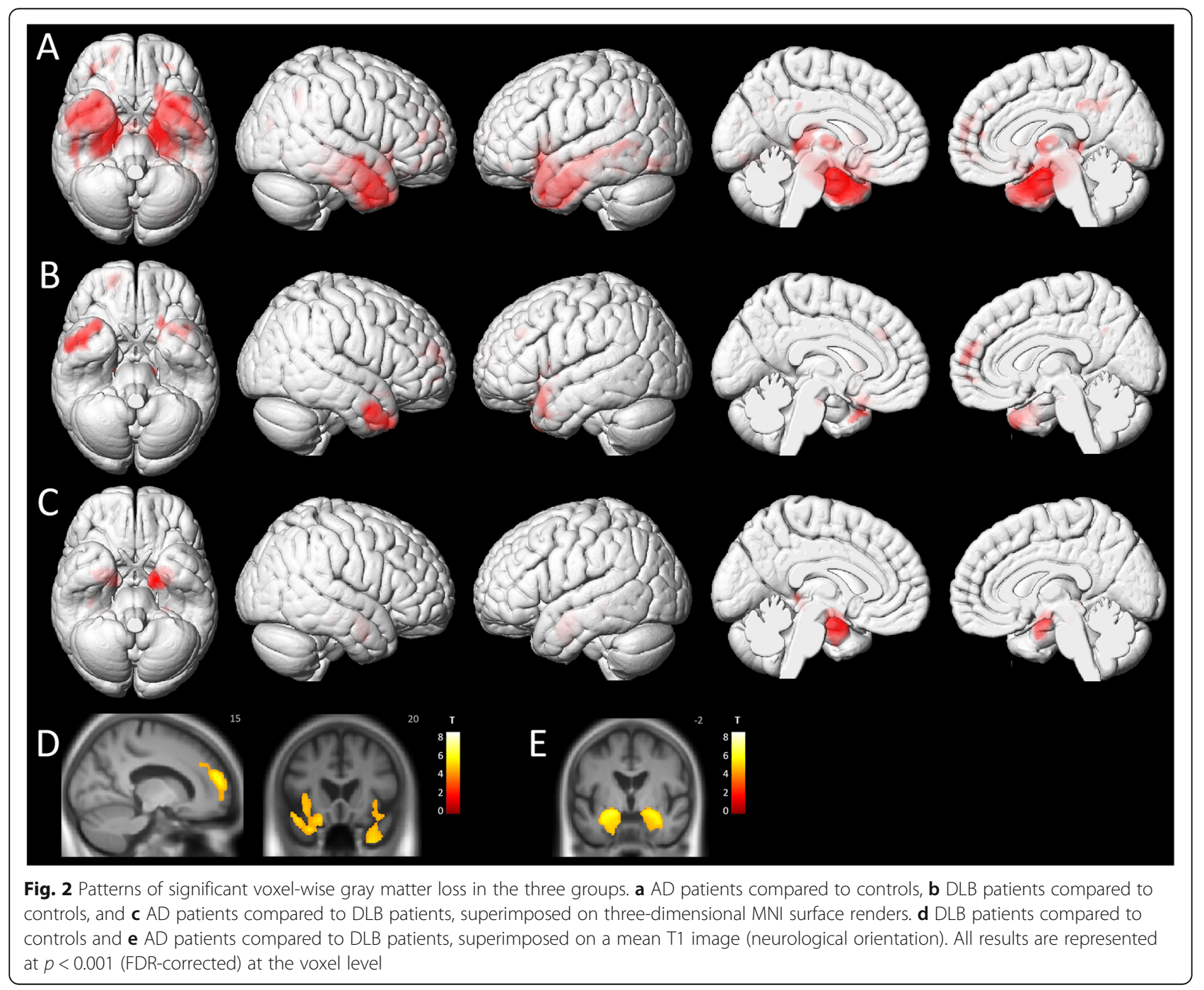


Table 2 Location and peak significance of clusters where gray matter volume was negatively correlated with fluctuation scores in the DLB group

\begin{tabular}{|c|c|c|c|c|c|c|c|}
\hline Anatomical region & Peak level $\left(p_{\text {unc }}\right)$ & Extent $(\mathrm{k})$ & $t$ & $z$ & $\mathrm{MNI} \mathrm{CO}$ & tes $(x, y, z)$ & \\
\hline Left inferior parietal lobule & $p<0.0001$ & 141 & 4.63 & 4.36 & -45 & -45 & 52.5 \\
\hline Left cerebellum & $p<0.0001$ & 165 & 4 & 3.82 & -9 & -52 & -32 \\
\hline Right cerebellum & $p<0.0001$ & 1212 & 3.97 & 3.79 & 42 & -54 & 26 \\
\hline Midbrain & $p<0.0001$ & 35 & 3.47 & 3.35 & -19.5 & -16.5 & -12 \\
\hline Right middle frontal gyrus (orbital part) & $p=0.0008$ & 15 & 3.27 & 3.17 & 46 & 51 & -4.5 \\
\hline Left superior frontal gyrus (orbital part) & $p=0.0009$ & 9 & 3.24 & 3.14 & -10.5 & 42 & -24 \\
\hline Right thalamus & $p=0.0027$ & 186 & 2.86 & 2.79 & 3 & -8 & 4 \\
\hline
\end{tabular}

No significant differences were found between the $\mathrm{AD}$ and control groups or between the DLB and AD groups at the set FDR-corrected threshold. At an uncorrected threshold of $p<0.05, \mathrm{AD}$ patients showed a connectivity decrease between several ROIs within the $\mathrm{SN}$ and within the DMN, and a higher connectivity between the MPFC of the DMN and the right RPFC of the $\mathrm{SN}$, compared to the control group. When comparing the $\mathrm{AD}$ and DLB groups $(p<0.01$ uncorrected), DLB patients had a weaker connectivity between two ROIs within the SN (left RPFC and left anterior insula $[\mathrm{AI}]$ ), and between ROIs of this network and ROIs of the DAN (right frontal eye field [FEF]), FPN (left and right LPFC), and DMN (precuneal cortex $[\mathrm{PCC}])$. Conversely, they showed a higher connectivity than $\mathrm{AD}$ patients between two ROIs within the DMN (right lateral parietal cortex [LP] and PCC) and between the left IPS of the DAN and the left LPFC of the FPN (see Fig. 3).

\section{Mean within- and between-network connectivity}

The DLB group and AD group showed a weaker mean functional connectivity within the SN compared to the control group $\left(T_{154}=2.96, p=0.0035\right.$ and $T_{154}=2.04$, $p=0.0430$, respectively). No other within-network and no between-network connectivity differences were found among the three groups.

\section{Association between FC measures and fluctuation scores}

In the DLB group, we found a positive correlation between patients' fluctuation scores and functional connectivity for three pairs of ROIs: left SN-RPFC and left DAN-FEF, left SN-AI and right DAN-FEF, right SN-AI and left thalamus. Fluctuation scores were also negatively correlated to the functional connectivity between the left SAL-RPFC and the DMN-MPFC, between the right thalamus and all the ROIs of the DMN (MPFC, left and right LP, PCC), between the right thalamus and two
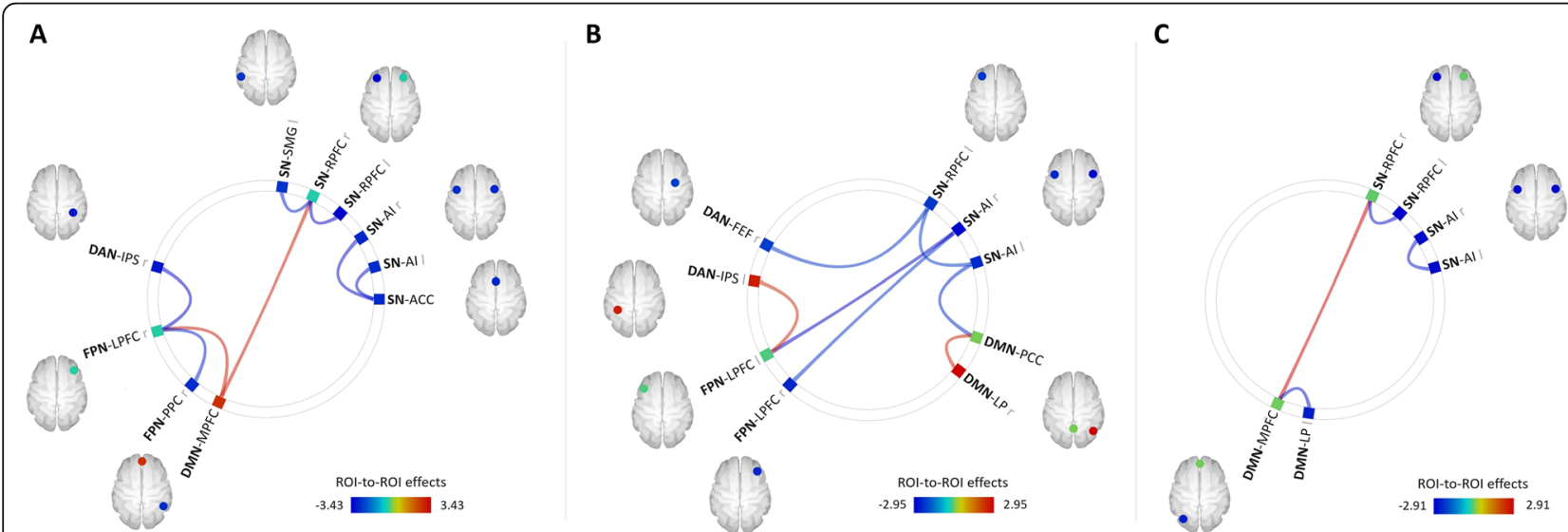

Fig. 3 Between-group differences in ROI-to-ROI functional connectivity. a DLB > controls, b DLB > AD, and c AD > controls. Abbreviations: ACC, anterior cingulate cortex; Al, anterior insula; DAN, dorsal attention network; DMN, default-mode network; FEF, frontal eye field; FPN, frontoparietal network; IPS, intraparietal sulcus; I, left; LP, lateral parietal cortex; LPFC, lateral prefrontal cortex; MPFC, medial prefrontal cortex; PCC, precuneal cortex; PPC, posterior parietal cortex; r, right; RPFC, rostral prefrontal cortex; SN, salience network; SMG, supramarginal gyrus. The results are represented respectively at $\mathbf{a} p_{\mathrm{FWE}}<0.05, \mathbf{b} p_{\mathrm{unc}}<0.01$, and Figure $\mathrm{S} 1 p_{\mathrm{unc}}<0.05$, at the ROl level 
ROIs of the FPN (left and right PPC), as well as between the left thalamus and the DMN-PCC (see Fig. 4).

\section{Discussion}

In the present study, we aimed to investigate the structural and functional changes in DLB compared with AD and normal aging and to examine their potential role in the emergence of the fluctuations characterizing these patients.

With regard to structural aspects, the results of group comparisons performed in our VBM analysis were in agreement with the existing literature. In the $\mathrm{AD}$ group compared to the control group, we found a typical pattern of medial temporal atrophy, as consistently described in previous studies [28]. In contrast, gray matter loss in the DLB group compared to controls was less extensive and consisted of focal bilateral clusters in the temporal lobes, insulae, and frontal lobes. The changes observed in temporal and frontal regions were consistent with the results of several earlier VBM studies [29, 30]. Similarly, diminished gray matter volumes in bilateral insulae were previously reported in DLB patients at both the dementia [31] and prodromal [32, 33] stages. The insular cortex was also shown to be particularly vulnerable to $\alpha$-synuclein pathology in these patients [34]. Unlike some previous studies [35-37], we did not find any significant gray matter loss in subcortical structures in the DLB group compared to the control group. This could be explained by the fact that the group comparisons in most of these studies were performed on chosen segmented subcortical structures rather than on the whole brain gray matter. Another possible explanation to this result is that our DLB patients were at early stages of the disease (prodromal and mild dementia stages), with a mean group MMSE of 25.6 indicating a very mild cognitive decline. Previous studies did not show subcortical gray matter loss in DLB patients at the prodromal stage [32,33], so it is possible that changes in these regions occur later in the disease course. To further explore the potential subcortical gray matter changes in our DLB group, we evaluated the differences between the DLB and control subjects with a more permissive threshold of $p<0.0001$ (uncorrected for multiple comparisons). The results are shown in Additional file 2: Figure S1. Our early-stage DLB patients showed trends of decreased gray matter volume in the thalamus and in

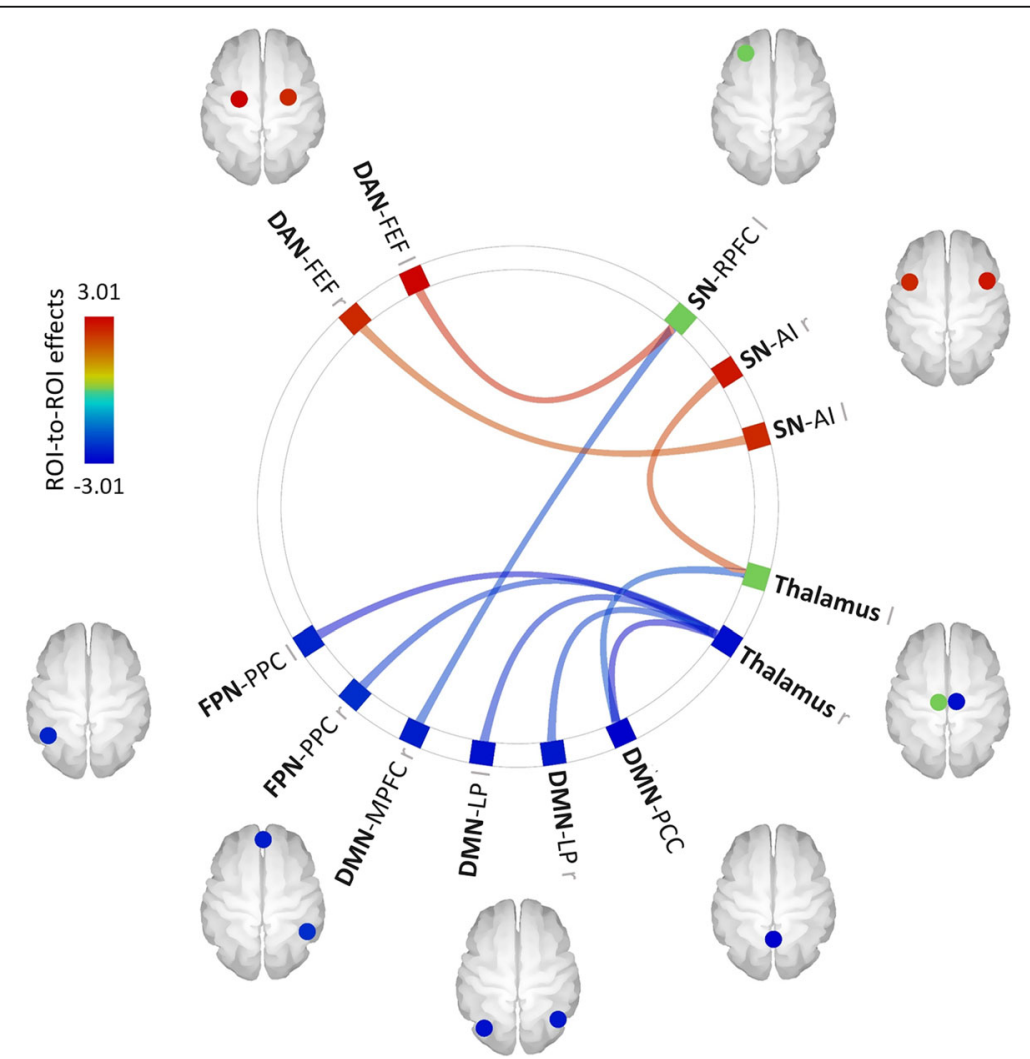

Fig. 4 Effect of fluctuation scores on functional connectivity in the DLB group. Abbreviations: Al, anterior insula; DAN, dorsal attention network; DMN, default-mode network; FEF, frontal eye field; FPN, frontoparietal network; I, left; LP, lateral parietal cortex; MPFC, medial prefrontal cortex; PCC, precuneus cortex; PPC, posterior parietal cortex; r, right; RPFC, rostral prefrontal cortex; SN, salience network. The results are represented at $p_{\text {unc }}<0.05$ at the ROI level 
the brainstem. These slight changes, which are not sufficiently marked to reach significance with an FDR correction, are consistent with previous studies showing atrophy in the same regions in these patients at the dementia stages [35, 36, 38] and tend to support the hypothesis of a later occurrence of subcortical volume loss in DLB. Furthermore, as pointed out in a recent review [39], the most significant subcortical changes reported in DLB patients in the literature relate to functional rather than structural imaging data. Finally, the comparison between the AD and DLB groups revealed a relative bilateral preservation of the medial temporal lobe structures in the latter, in line with a number of existing data reviewed by Surendranathan [40].

Among these results, gray matter losses found in the insulae and frontal regions in the DLB group may be relevant with regard to fluctuations. The insula is a complex cortical region participating in a wide range of cognitive, emotional, somatosensory, and visceral functions [41], and its anterior division forms a major node of the $\mathrm{SN}$. Considering the critical role of the $\mathrm{SN}$ for the switching between default-mode and task-positive networks [42] and the well-known involvement of frontal regions in attentional-executive processes, such atrophic patterns could potentially participate in fluctuations in DLB patients.

However, when investigating more directly the correlation between patients' fluctuation scores and gray matter volume in the DLB group, we found a weak negative correlation in the left inferior parietal lobule, the left and right cerebellum, the midbrain, the bilateral prefrontal cortex, and to a lesser extent, the right thalamus. These regions did not correspond to those showing gray matter loss in DLB patients compared to control subjects, which suggests that the relative atrophy of frontal and insular cortices may not be directly involved in the occurrence of fluctuations. Moreover, the effect of fluctuation scores on regional gray matter volume seemed to follow a particular pattern, as the corresponding brain regions were part of the cholinergic system. This system plays an important role in attention and conscious awareness $[43,44]$ and was shown to be more affected in DLB than in AD $[45,46]$. The disturbances identified in DLB patients notably include deficits in ChAT [47] and AChE [48] and changes in nicotinic and muscarinic receptors density [49-51] compared to $\mathrm{AD}$ patients and healthy elderly subjects. Several studies reviewed by Aarsland et al. [52] also demonstrated that cholinesterase inhibitors have a positive effect on cognition and neuropsychiatric symptoms in DLB patients, including fluctuating attention, unresponsiveness, and daytime somnolence [53]. Similarly, neuropathological analyses comparing fluctuating and non-fluctuating DLB patients reported significant cholinergic impairments in thalamic areas in the former [54]. Taken together, these findings suggest that cholinergic impairments may be a determinant factor in the etiology of cognitive fluctuations. In the abovementioned regions where gray matter volume was found to be negatively correlated to fluctuation scores, but which are not significantly atrophied in DLB patients compared to controls, it is possible that cholinergic synaptic dysfunction may cause microstructural impairments (such as a reduced synaptic density) without frank neuronal loss, thus only leading to subtle volume reductions. Indeed, other neurotransmitter systems involved in attention and arousal, such as noradrenergic or dopaminergic systems, suffer neuronal loss in DLB $[55,56]$ and could therefore also contribute to a lesser degree to the pathogenesis of this symptom.

Globally, the results of our VBM analysis thus suggest that fluctuations might be more related to microstructural (i.e., slight volume reductions secondary to a loss of synapses) or functional changes, rather than macrostructural modifications.

To investigate the potential spatial correspondence between structural and functional disturbances related to fluctuations in the DLB group, the thalami and midbrain were added as complementary ROIs for the subsequent functional connectivity analysis (the inferior parietal and prefrontal regions did not need to be added as they already corresponded to key nodes of the preselected functional networks).

The second part of our study focused on functional connectivity aspects. At the network level, when compared to the control group, the DLB patients showed significant decreases in functional connectivity within the $\mathrm{SN}$ and within the FPN, and between ROIs of the FPN and DAN. In contrast, they showed an increased connectivity between ROIs of the DMN and ROIs of both the $\mathrm{SN}$ and the FPN. In the AD group, the changes relative to the control group were globally weaker than those distinguishing the DLB group from the control group. They showed a decreased functional connectivity within the SN but also within the DMN, along with an increased connectivity between ROIs from those two networks. Finally, when comparing directly the two dementia groups, DLB patients showed a weaker ROI-toROI functional connectivity than AD patients within the $\mathrm{SN}$, and between this network and the DAN, the DMN and the FPN. Conversely, the DLB group had a stronger connectivity than the $\mathrm{AD}$ group within the $\mathrm{DMN}$, and between ROIs of the DAN and FPN. Additional measurements confirmed a weaker mean connectivity within the $\mathrm{SN}$ in the DLB and AD groups compared with the control group.

These results tend to identify specific patterns of functional connectivity disturbances in DLB and AD. Both groups showed a decreased connectivity within the SN 
compared to healthy elderly subjects, which was accompanied by an increased connectivity between the right RPFC of the SN and the MPFC of the DMN. This increase could constitute a compensation process, in order to maintain an effective coupling between the two networks despite the functional disruptions in the SN. In the DLB group, the increased connectivity between the FPN-LPFC and the DMN-MPFC could similarly be an attempt to compensate for the connectivity disturbances within and between attentional networks. But beyond these similarities, the two groups also revealed distinct profiles of impairments, as SN disturbances appeared to co-occur primarily with attentional network disruptions in the DLB group, while they rather coincided with DMN disruptions in the AD group. In AD patients, this decrease in DMN functional connectivity is a consistently reported feature in previous studies, irrespective of the analytical approach used [57]. Regarding the SN, the literature is less consistent: some authors reported an increased functional connectivity in this network in $\mathrm{AD}$ compared to normal aging $[58,59]$, while others found it was decreased [60,61]. This could be explained by methodological differences between the analyses (ICA versus seed-based approaches). However, Brier et al. [62] provided another possible explanation by showing in a larger cohort $(n=124)$ that functional connectivity within the $\mathrm{SN}$ was increased in $\mathrm{AD}$ patients at a very early stage of the disease (clinical dementia rating [CDR] $0.5)$, but then decreased at a later stage (CDR 1$)$.

In DLB, our findings are similar to those of Lowther et al. [63], who found a decreased connectivity in the salience and executive control networks compared with AD patients and healthy subjects. The fact that we found decreases specifically within the FPN and the SN and between the FPN and the DAN, may provide some clues about the occurrence of fluctuations and attention deficits $[1,3]$ in DLB patients, considering the particular interaction between these networks. The FPN and DAN show high activity during externally directed attentional focus and are anticorrelated with the DMN, which is activated during unfocused, internally focused, or exploratory states $[64,65]$. The coupling of these networks, which allows an effective switching between distinct attentional states in response to both internal and environmental stimuli, was shown to be triggered by the activity of the SN $[42,66]$. The functional connectivity disruptions that we observed in the DLB group may lead to disturbed transitions from one network configuration (or cognitive state) to another and could thus participate in the etiology of fluctuations.

The multiple regression analysis revealed that the severity of fluctuations in DLB patients was positively correlated to the functional connectivity of the $\mathrm{SN}$ with the DAN and the left thalamus and negatively correlated with the connectivity between the $\mathrm{SN}$ and the $\mathrm{DMN}$ and between the right thalamus and both the FPN and the DMN.

First, this suggests that fluctuations could arise from an imbalance of the external connectivity of the $\mathrm{SN}$, maybe triggered primarily by the disconnections between the different ROIs within this network, as observed in the group comparisons. The negative correlation between fluctuation scores and functional connectivity between the $\mathrm{SN}$ and DMN is striking because it involves the RPFC of the SN and the MPFC of the DMN, a pair of ROIs showing a hyperconnectivity (but with a different lateralization) in DLB patients compared to healthy subjects, which we interpreted as a compensation process. In agreement with this hypothesis, greater fluctuations in DLB patients may thus be linked to a failure of this compensation. Second, these findings tend to confirm the involvement of thalamic functional disturbances in the etiology of fluctuations in DLB patients, as suggested by previous studies $[10,18]$. The thalamus has strong functional connections with cortical nodes of the $\mathrm{SN}$, especially the anterior insula hub, and participates with it in a large-scale network integrating interoceptive inputs with cognitive inputs from other networks and generating viscero-autonomic, emotional, and cognitive processes in response to salient stimuli [67, 68]. Moreover, the functional and structural connectivity of the thalamus with the DMN $[69,70]$ and the frontoparietal regions [71] was shown to be crucial for consciousness. The negative correlation between thalamic functional connectivity and fluctuation scores in the DLB group therefore seems concordant with the clinical characteristics of this symptom. Furthermore, this correlation could also be consistent with the results of our VBM analysis showing a negative correlation between fluctuation scores and gray matter volume in several cholinergic regions. Indeed, as mentioned by Peraza et al. [72], loss of cholinergic function may impair the ability of the cholinergic system to inhibit intracortical short-range functional connections, which is the mechanism by which the brain can enhance thalamocortical interactions in response to external stimuli $[73,74]$. It is thus possible that both phenomena may contribute to the occurrence of fluctuations in DLB patients. Alternatively, thalamic functional disturbances could also be a consequence of cholinergic function deficits.

Overall, our results indicate that fluctuations in DLB patients may derive more directly from functional connectivity disturbances than from clear structural impairments. This is concordant with recent data supporting the hypothesis that DLB is a primary synaptopathy [75], in which the accumulation of phosphorylated $\alpha$-synuclein in the form of small aggregates may cause synaptic dysfunction and loss that occur prior to Lewy body formation and neuronal loss and that are closely related to cognitive impairment. 
This study has some potential limitations. First, DLB and $\mathrm{AD}$ patients were diagnosed based on clinical assessment, rather than a post-mortem pathological validation. However, diagnosis was made by experienced geriatricians, using standardized criteria with a high specificity confirmed by autopsy findings [76], and all participants were followed longitudinally. Second, fluctuations are a complex symptom encompassing attentional, cognitive, and alertness aspects that are mixed in the score obtained from the Mayo Fluctuation Scale, making the interpretation of the correlations with MRI measures more difficult. These results thus need to be treated with caution.

\section{Conclusions}

Using both structural and functional MRI data, we found that functional connectivity changes, rather than significant gray matter loss, could play a role in the emergence of fluctuations in DLB patients. Notably, fluctuations in the DLB group appeared to be related to disturbances of the external functional connectivity of the salience network (responsible for switching between the default mode network and attentional executive networks), which may lead to less relevant transitions from one cognitive state to another in response to internal and environmental stimuli. Higher fluctuation scores were also related to a lower thalamic functional connectivity, suggesting that the thalamus could be a key region for the occurrence of this symptom. More globally, our results underline the interest of considering large-scale brain networks to investigate the neural bases of DLB core symptoms such as fluctuations.

\section{Supplementary information}

The online version of this article (https://doi.org/10.1186/s13195-019-0575-z) contains supplementary material, which is available to authorized users.

Additional file 1: Table S1. MNI coordinates and anatomical labels of the regions of interest used in the functional connectivity analysis

Additional file 2: Figure S1. Pattern of voxel-wise gray matter loss in DLB patients compared with healthy elderly subjects at a more permissive significance threshold ( $p<0.0001$ uncorrected for multiple comparisons). Abbreviations: BC: brainstem, Thal: thalamus

\section{Abbreviations}

ACC: Anterior cingulate cortex; AD: Alzheimer's disease; Al: Anterior insula; ANOVA: Analysis of variance; BOLD: Blood oxygen level-dependent; CDR: Clinical dementia rating; CM2R: Centre Mémoire de Ressources et de Recherche; CPP: Comité de protection des personnes; DAN: Dorsal attention network; DARTEL: Diffeomorphic anatomical registration through exponentiated lie algebra; DLB: Dementia with Lewy bodies; DMN: Defaultmode network; FDR: False discovery rate; FEF: Frontal eye field; FPN: Frontoparietal network; FWE: Family-wise error; HC: Healthy controls; HUS: University Hospital of Strasbourg; ICA: Independent component analysis; IPS: Intraparietal sulcus; LP: Lateral parietal cortex; LPFC: Lateral prefrontal cortex; MNI: Montreal Neurological Institute; MPFC: Medial prefrontal cortex; MPRAGE: Magnetization-prepared rapid gradient-echo; MRI: Magnetic resonance imaging; PCC: Precuneal cortex; PPC: Posterior parietal cortex; ROI: Region of interest; RPFC: Rostral prefrontal cortex; SI: Substantia innominata; SMG: Supramarginal gyrus; SN: Salience network;
SPECT: Single-photon emission computed tomography; SPM: Statistical Parametric Mapping; VBM: Voxel-based morphometry

\section{Acknowledgements}

We thank the patients and control subjects for their participation. We are grateful to Jean-Paul Armspach and Corinne Marrer from the "Plateforme IRIS, Faculté de Médecine, Université de Strasbourg" for MRI acquisition; to Thimothée Albasser, Mathias Bilger, Laure Di Bitonto, Emmanuelle Ehrhard, Catherine Kleitz, Nadine Longato, Laetitia Monjoin, Géraldine Heim, and Natacha Vogt for conducting neuropsychological assessments; to Marion Sourty and Daniel Roquet for allowing us to use their preprocessing pipeline; to Mary Mondino for her help with programming; to Julien Lamy for his advice and comments; to Anne Botzung and Lucie Rauch for their help with organization; and to Nick Barton for language editing.

\section{Funding}

This study was funded by Appel à Projet Interne (API) of the University Hospital of Strasbourg, Alsace Alzheimer 67, Fondation Université de Strasbourg, and Projet Hospitalier de Recherche Clinique (PHRC) inter-régional (IDRCB 2012-A00992-41), and a ministerial PhD scholarship.

\section{Availability of data and materials}

The datasets used and/or analyzed during the present study are available from the corresponding author on reasonable request.

\section{Authors' contributions}

EC participated in the design of the study, performed the MRI preprocessing and the statistical analysis, interpreted the data, and drafted the manuscript. VN participated in the design of the study and performed the MRI preprocessing. PLDS optimized the MRI sequences. CD, NP, CM, PA, CMH, and $B C$ examined the patients and diagnosed patients with DLB and AD. FB conceived of the study, participated in its design and coordination, examined patients, diagnosed patients with DLB and AD, interpreted the data, and revised the manuscript. All authors read and approved the final manuscript.

\section{Ethics approval and consent to participate}

This research was approved by the local ethics committee ("Comité de Protection des Personnes Strasbourg Est IV"). All participants provided written informed consent to participate.

\section{Consent for publication}

Written informed consent was obtained from the participants for publication of their individual details and accompanying images in this manuscript. The consent form is held by the authors' institution and is available for review by the Editor-in-Chief.

\section{Competing interests}

The authors declare that they have no competing interests.

\section{Author details}

${ }^{1}$ ICube Laboratory UMR 7357 and FMTS (Fédération de Médecine Translationnelle de Strasbourg), Team IMIS, University of Strasbourg and CNRS, Strasbourg, France. ${ }^{2}$ CM2R (Research and Resources Memory Centre), Geriatrics Department, University Hospitals of Strasbourg, Geriatric Day Hospital and Neuropsychology Unit, Strasbourg, France. ${ }^{3}$ INSERM Centre d'Investigation Clinique 1434, University Hospitals of Strasbourg, Strasbourg, France. ${ }^{4}$ General Hospital Centre, Geriatrics Department, CM2R, Geriatric Day Hospital, Colmar, France.

Received: 1 May 2019 Accepted: 23 December 2019 Published online: 06 January 2020

\section{References}

1. McKeith IG, Boeve BF, Dickson DW, Halliday G, Taylor J-P, Weintraub D, et al. Diagnosis and management of dementia with Lewy bodies: Fourth consensus report of the DLB Consortium. Neurology. 2017;89(1):88-100.

2. McKeith IG, Dickson DW, Lowe J, Emre M, O'Brien JT, Feldman H, et al. Diagnosis and management of dementia with Lewy bodies: third report of the DLB Consortium. Neurology. 2005;65(12):1863-72. 
3. Ballard C, O'Brien J, Gray A, Cormack F, Ayre G, Rowan E, et al. Attention and fluctuating attention in patients with dementia with Lewy bodies and Alzheimer disease. Arch Neurol. 2001;58(6):977-82.

4. Mainland BJ, Herrmann N, Mallya S, Fiocco AJ, Sin G-L, Shulman Kl, et al. Cognitive fluctuations and cognitive test performance among institutionalized persons with dementia. Am J Alzheimers Dis Other Dement. 2017;32(7):393-400.

5. Zweig YR, Galvin JE. Lewy body dementia: the impact on patients and caregivers. Alzheimers Res Ther. 2014;6(2):21.

6. Ferman TJ, Smith GE, Boeve BF, Ivnik RJ, Petersen RC, Knopman D, et al. DLB fluctuations: specific features that reliably differentiate DLB from $A D$ and normal aging. Neurology. 2004;62(2):181-7.

7. Walker MP, Ayre GA, Cummings JL, Wesnes K, McKeith IG, O'Brien JT, et al. The clinician assessment of fluctuation and the one day fluctuation assessment scale. Two methods to assess fluctuating confusion in dementia. Br J Psychiatry J Ment Sci. 2000;177:252-6.

8. Van Dyk K, Towns S, Tatarina O, Yeung P, Dorrejo J, Zahodne LB, et al. Assessing fluctuating cognition in dementia diagnosis: Interrater reliability of the clinician assessment of fluctuation. Am J Alzheimers Dis Other Dement. 2016;31(2):137-43.

9. Bradshaw J, Saling M, Hopwood M, Anderson V, Brodtmann A. Fluctuating cognition in dementia with Lewy bodies and Alzheimer's disease is qualitatively distinct. J Neurol Neurosurg Psychiatry. 2004;75(3):382-7.

10. Delli Pizzi S, Franciotti R, Taylor J-P, Thomas A, Tartaro A, Onofrj M, et al. Thalamic involvement in fluctuating cognition in dementia with Lewy bodies: magnetic resonance evidences. Cereb Cortex. 2015;25(10):3682-9.

11. Watson R, Colloby SJ, Blamire AM, Wesnes KA, Wood J, O'Brien JT. Does attentional dysfunction and thalamic atrophy predict decline in dementia with Lewy bodies? Parkinsonism Relat Disord. 2017;45:69-74.

12. Colloby SJ, Elder GJ, Rabee R, O'Brien J, Taylor J-P. Structural grey matter changes in the substantia innominata in Alzheimer's disease and dementia with Lewy bodies: a DARTEL-VBM study. Int J Geriatr Psychiatry. 2017;32(6):615-23.

13. Taylor J-P, Colloby SJ, McKeith IG, O'Brien JT. Covariant perfusion patterns provide clues to the origin of cognitive fluctuations and attentional dysfunction in dementia with Lewy bodies. Int Psychogeriatr. 2013;25(12): 1917-28.

14. Peraza LR, Kaiser M, Firbank M, Graziadio S, Bonanni L, Onofrj M, et al. fMRI resting state networks and their association with cognitive fluctuations in dementia with Lewy bodies. Neuroimage Clin. 2014;4:558-65.

15. Bonanni L, Thomas A, Tiraboschi P, Perfetti B, Varanese S, Onofrj M. EEG comparisons in early Alzheimer's disease, dementia with Lewy bodies and Parkinson's disease with dementia patients with a 2-year follow-up. Brain. 2008;131(3):690-705

16. Bonanni L, Perfetti B, Bifolchetti S, Taylor J-P, Franciotti R, Parnetti $L$, et al. Quantitative electroencephalogram utility in predicting conversion of mild cognitive impairment to dementia with Lewy bodies. Neurobiol Aging. 2015;36(1):434-45.

17. Stylianou M, Murphy N, Peraza LR, Graziadio S, Cromarty R, Killen A, et al. Quantitative electroencephalography as a marker of cognitive fluctuations in dementia with Lewy bodies and an aid to differential diagnosis. Clin Neurophysiol. 2018;129(6):1209-20.

18. O'Brien JT, Firbank MJ, Mosimann UP, Burn DJ, McKeith IG. Change in perfusion, hallucinations and fluctuations in consciousness in dementia with Lewy bodies. Psychiatry Res Neuroimaging. 2005;139(2):79-88.

19. Franciotti R, Falasca NW, Bonanni L, Anzellotti F, Maruotti V, Comani S, et al. Default network is not hypoactive in dementia with fluctuating cognition: an Alzheimer disease/dementia with Lewy bodies comparison. Neurobiol Aging. 2013;34(4):1148-58

20. Dubois B, Feldman HH, Jacova C, DeKosky ST, Barberger-Gateau P Cummings J, et al. Research criteria for the diagnosis of Alzheimer's disease: revising the NINCDS-ADRDA criteria. Lancet Neurol. 2007;6(8):734-46.

21. Kemp J, Philippi N, Phillipps C, Demuynck C, Albasser T, Martin-Hunyadi C, et al. Cognitive profile in prodromal dementia with Lewy bodies. Alzheimers Res Ther. 2017;9(1):19.

22. Good CD, Johnsrude IS, Ashburner J, Henson RN, Friston KJ, Frackowiak RS A voxel-based morphometric study of ageing in 465 normal adult human brains. Neuroimage. 2001;14(1 Pt 1):21-36.

23. Ashburner J, Friston KJ. Unified segmentation. Neuroimage. 2005;26(3):839-51.

24. Muschelli J, Nebel MB, Caffo BS, Barber AD, Pekar JJ, Mostofsky SH. Reduction of motion-related artifacts in resting state fMRI using aCompCor. Neurolmage. 2014;96:22-35.
25. Friston KJ, Holmes AP, Worsley KJ, Poline J-P, Frith CD, Frackowiak RSJ. Statistical parametric maps in functional imaging: a general linear approach. Hum Brain Mapp. 1994;2(4):189-210.

26. Whitfield-Gabrieli S, Nieto-Castanon A. Conn: a functional connectivity toolbox for correlated and anticorrelated brain networks. Brain Connectivity. 2012;2(3):125-41.

27. Desikan RS, Ségonne F, Fischl B, Quinn BT, Dickerson BC, Blacker D, et al. An automated labeling system for subdividing the human cerebral cortex on MRI scans into gyral based regions of interest. Neurolmage. 2006;31(3):968-80.

28. Scheltens $P$, Leys D, Barkhof F, Huglo D, Weinstein HC, Vermersch $P$, et al. Atrophy of medial temporal lobes on MRI in «probable » Alzheimer's disease and normal ageing: diagnostic value and neuropsychological correlates. J Neurol Neurosurg Psychiatry. 1992; 55(10):967-72.

29. Watson R, O'Brien JT, Barber R, Blamire AM. Patterns of gray matter atrophy in dementia with Lewy bodies: a voxel-based morphometry study. Int Psychogeriatr. 2012;24(4):532-40.

30. Burton EJ, Karas G, Paling SM, Barber R, Williams ED, Ballard CG, et al. Patterns of cerebral atrophy in dementia with Lewy bodies using voxelbased morphometry. Neurolmage. 2002;17(2):618-30.

31. Zhong J, Pan P, Dai Z, Shi H. Voxelwise meta-analysis of gray matter abnormalities in dementia with Lewy bodies. Eur J Radiol. 2014;83(10): 1870-4.

32. Blanc F, Colloby SJ, Cretin B, de Sousa PL, Demuynck C, O'Brien JT, et al. Grey matter atrophy in prodromal stage of dementia with Lewy bodies and Alzheimer's disease. Alzheimers Res Ther. 2016:8(1):31.

33. Roquet $D$, Noblet $V$, Anthony $P$, Philippi N, Demuynck C, Cretin B, et al. Insular atrophy at the prodromal stage of dementia with Lewy bodies: a VBM DARTEL study. Sci Rep. 2017;7(1):9437.

34. Fathy YY, Jonker AJ, Oudejans E, de Jong FJJ, van Dam A-MW, Rozemuller AJM, et al. Differential insular cortex subregional vulnerability to a-synuclein pathology in Parkinson's disease and dementia with Lewy bodies. Neuropathol Appl Neurobiol. 2019;45(3):262-77.

35. Watson R, Colloby SJ, Blamire AM, O'Brien JT. Subcortical volume changes in dementia with Lewy bodies and Alzheimer's disease. A comparison with healthy aging. Int Psychogeriatr. 2016;28(4):529-36.

36. Whitwell JL, Weigand SD, Shiung MM, Boeve BF, Ferman TJ, Smith GE, et al. Focal atrophy in dementia with Lewy bodies on MRI: a distinct pattern from Alzheimer's disease. Brain. 2007;130(Pt 3):708-19.

37. Cousins DA, Burton EJ, Burn D, Gholkar A, McKeith IG, O'Brien JT. Atrophy of the putamen in dementia with Lewy bodies but not Alzheimer's disease: an MRI study. Neurology. 2003;61(9):1191-5.

38. Shimizu S, Hanyu H, Hirao K, Sato T, Iwamoto T, Koizumi K. Value of analyzing deep gray matter and occipital lobe perfusion to differentiate dementia with Lewy bodies from Alzheimer's disease. Ann Nucl Med déc. 2008;22(10):911-6.

39. Watson R, Colloby SJ. Imaging in dementia with Lewy bodies: an overview. J Geriatr Psychiatry Neurol. 2016;29(5):254-60.

40. Surendranathan A, O'Brien JT. Clinical imaging in dementia with Lewy bodies. Evid Based Mental Health. 2018;21(2):61-5.

41. Nieuwenhuys R. The insular cortex: A review. In: Hofman MA, Falk D, editors. Progress in brain research: evolution of the primate brain; vol. 195: Elsevier; 2012. p. 123-63.

42. Sridharan D, Levitin DJ, Menon V. A critical role for the right fronto-insular cortex in switching between central-executive and default-mode networks. PNAS. 2008;105(34):12569-74.

43. Perry E, Walker M, Grace J, Perry R. Acetylcholine in mind: a neurotransmitter correlate of consciousness? Trends Neurosci. 1999;22(6):273-80.

44. Klinkenberg I, Sambeth A, Blokland A. Acetylcholine and attention. Behav Brain Res. 2011;221(2):430-42.

45. Tiraboschi P, Hansen LA, Alford M, Merdes A, Masliah E, Thal LJ, et al. Early and widespread cholinergic losses differentiate dementia with Lewy bodies from Alzheimer disease. Arch Gen Psychiatry. 2002;59(10):946-51.

46. Francis PT, Perry EK. Cholinergic and other neurotransmitter mechanisms in Parkinson's disease, Parkinson's disease dementia, and dementia with Lewy bodies. Mov Disord. 2007;22(S17):S351-7.

47. Tiraboschi P, Hansen LA, Alford M, Sabbagh MN, Schoos B, Masliah E, et al. Cholinergic dysfunction in diseases with Lewy bodies. Neurology. 2000; 54(2):407-11.

48. Shimada H, Hirano S, Sinotoh H, Ota T, Tanaka N, Sato K, et al. Dementia with Lewy bodies can be well-differentiated from Alzheimer's disease by 
measurement of brain acetylcholinesterase activity-a [11C]MP4A PET study. Int J Geriatr Psychiatry. 2015;30(11):1105-13.

49. Perry EK, Morris CM, Court JA, Cheng A, Fairbairn AF, McKeith IG, et al. Alteration in nicotine binding sites in Parkinson's disease, Lewy body dementia and Alzheimer's disease: possible index of early neuropathology. Neuroscience. 1995;64(2):385-95.

50. Piggott MA, Owens J, O'Brien J, Colloby S, Fenwick J, Wyper D, et al. Muscarinic receptors in basal ganglia in dementia with Lewy bodies, Parkinson's disease and Alzheimer's disease. J Chem Neuroanatomy. 2003; 25(3):161-73.

51. O'Brien JT, Colloby SJ, Pakrasi S, Perry EK, Pimlott SL, Wyper DJ, et al. Nicotinic alpha4beta2 receptor binding in dementia with Lewy bodies using 123I-5IA-85380 SPECT demonstrates a link between occipital changes and visual hallucinations. Neuroimage. 2008;40(3):1056-63.

52. Aarsland D, Mosimann UP, McKeith IG. Role of cholinesterase inhibitors in Parkinson's disease and dementia with Lewy bodies. J Geriatr Psychiatry Neurol. 2004;17(3):164-71.

53. Wesnes KA, McKeith IG, Ferrara R, Emre M, Ser TD, Spano PF, et al. Effects of rivastigmine on cognitive function in dementia with Lewy bodies: a randomised placebo-controlled international study using the cognitive drug research computerised assessment system. DEM. 2002;13(3):183-92.

54. Pimlott SL, Piggott M, Ballard C, McKeith I, Perry R, Kometa S, et al. Thalamic nicotinic receptors implicated in disturbed consciousness in dementia with Lewy bodies. Neurobiol Dis. 2006;21(1):50-6.

55. Szot P, White SS, Greenup JL, Leverenz JB, Peskind ER, Raskind MA. Compensatory changes in the noradrenergic nervous system in the locus ceruleus and hippocampus of postmortem subjects with Alzheimer's disease and dementia with Lewy bodies. J Neurosci. 2006;26(2):467-78.

56. O'Brien JT, Colloby S, Fenwick J, Williams ED, Firbank M, Burn D, et al. Dopamine transporter loss visualized with FP-CIT SPECT in the differential diagnosis of dementia with Lewy bodies. Arch Neurol. 2004;61(6):919-25.

57. Badhwar A, Tam A, Dansereau C, Orban P, Hoffstaedter F, Bellec P. Restingstate network dysfunction in Alzheimer's disease: a systematic review and meta-analysis. Alzheimers Dement. 2017;8:73-85.

58. Zhou J, Greicius MD, Gennatas ED, Growdon ME, Jang JY, Rabinovici GD, et al. Divergent network connectivity changes in behavioural variant frontotemporal dementia and Alzheimer's disease. Brain. 2010;133(5):1352-67.

59. Balthazar MLF, Pereira FRS, Lopes TM, da Silva EL, Coan AC, Campos BM et al. Neuropsychiatric symptoms in Alzheimer's disease are related to functional connectivity alterations in the salience network. Hum Brain Mapp. 2014;35(4):1237-46.

60. Wang Z, Xia M, Dai Z, Liang X, Song H, He Y, et al. Differentially disrupted functional connectivity of the subregions of the inferior parietal lobule in Alzheimer's disease. Brain Struct Funct. 2015;220(2):745-62.

61. Xie C, Bai F, Yu H, Shi Y, Yuan Y, Chen G, et al. Abnormal insula functional network is associated with episodic memory decline in amnestic mild cognitive impairment. Neuroimage. 2012;63(1):320-7.

62. Brier MR, Thomas JB, Snyder AZ, Benzinger TL, Zhang D, Raichle ME, et al. Loss of intranetwork and internetwork resting state functional connections with Alzheimer's disease progression. J Neurosci. 2012;32(26):8890-9.

63. Lowther ER, O'Brien JT, Firbank MJ, Blamire AM. Lewy body compared with Alzheimer dementia is associated with decreased functional connectivity in resting state networks. Psychiatry Res Neuroimaging. 2014;223(3):192-201.

64. Spreng RN, Stevens WD, Chamberlain JP, Gilmore AW, Schacter DL. Default network activity, coupled with the frontoparietal control network, supports goal-directed cognition. Neurolmage. 2010;53(1):303-17.

65. Hellyer PJ, Shanahan M, Scott G, Wise RJS, Sharp DJ, Leech R. The control of global brain dynamics: opposing actions of frontoparietal control and default mode networks on attention. J Neurosci. 2014;34(2):451-61.

66. Goulden N, Khusnulina A, Davis NJ, Bracewell RM, Bokde AL, McNulty JP, et al. The salience network is responsible for switching between the default mode network and the central executive network: replication from DCM. Neuroimage. 2014;99:180-90.

67. Zhou J, Seeley WW. Network dysfunction in Alzheimer's disease and frontotemporal dementia: implications for psychiatry. Biol Psychiatry. 2014; 75(7):565-73.

68. Uddin LQ. Salience processing and insular cortical function and dysfunction. Nat Rev Neurosci. 2015;16(1):55-61.

69. Boly M, Tshibanda L, Vanhaudenhuyse A, Noirhomme Q, Schnakers C, Ledoux $\mathrm{D}$, et al. Functional connectivity in the default network during resting state is preserved in a vegetative but not in a brain dead patient. Hum Brain Mapp. 2009;30(8):2393-400.

70. Fernández-Espejo D, Soddu A, Cruse D, Palacios EM, Junque C, Vanhaudenhuyse A, et al. A role for the default mode network in the bases of disorders of consciousness. Ann Neurology. 2012;72(3):335-43.

71. Crone JS, Soddu A, Höller Y, Vanhaudenhuyse A, Schurz M, Bergmann J, et al. Altered network properties of the fronto-parietal network and the thalamus in impaired consciousness. Neurolmage: Clinical. 2014;4:240-8.

72. Peraza LR, Taylor J-P, Kaiser M. Divergent brain functional network alterations in dementia with Lewy bodies and Alzheimer's disease. Neurobiol Aging. 2015a;36(9):2458-67.

73. Picciotto MR, Higley MJ, Mineur YS. Acetylcholine as a neuromodulator: cholinergic signaling shapes nervous system function and behavior. Neuron. 2012;76(1):116-29.

74. Lucas-Meunier E, Fossier P, Baux G, Amar M. Cholinergic modulation of the cortical neuronal network. Pflugers Arch - Eur J Physiol. 2003;446(1):17-29.

75. Colom-Cadena M, Pegueroles J, Herrmann AG, Henstridge CM, Muñoz L, Querol-Vilaseca M, et al. Synaptic phosphorylated a-synuclein in dementia with Lewy bodies. Brain. 2017;140(12):3204-14.

76. McKeith IG, Ballard CG, Perry RH, Ince PG, O'Brien JT, Neill D, et al. Prospective validation of consensus criteria for the diagnosis of dementia with Lewy bodies. Neurology. 2000;54(5):1050-8.

\section{Publisher's Note}

Springer Nature remains neutral with regard to jurisdictional claims in published maps and institutional affiliations.
Ready to submit your research? Choose BMC and benefit from:

- fast, convenient online submission

- thorough peer review by experienced researchers in your field

- rapid publication on acceptance

- support for research data, including large and complex data types

- gold Open Access which fosters wider collaboration and increased citations

- maximum visibility for your research: over $100 \mathrm{M}$ website views per year

At BMC, research is always in progress.

Learn more biomedcentral.com/submissions 